Vol. 2 • No. 7 • July • 2014
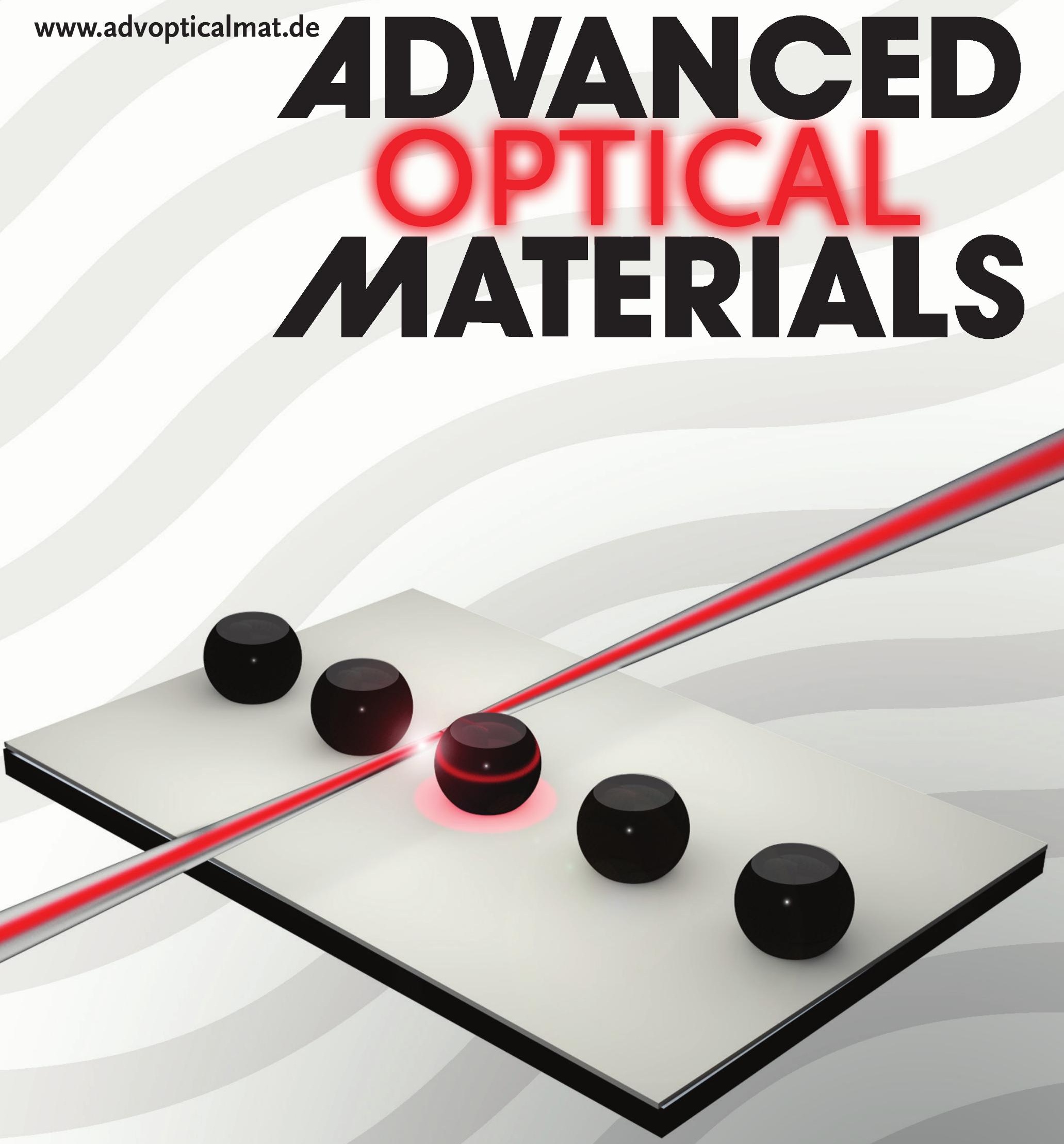


\title{
A New Route for Fabricating On-Chip Chalcogenide Microcavity Resonator Arrays
}

\author{
Ozan Aktas, Erol Ozgur, Osama Tobail, Mehmet Kanik, Ersin Huseyinoglu, \\ and Mehmet Bayindir*
}

The development of strategies for mass production and multiple integration of optical microresonators in photonic circuits has been a subject of intense research, aiming to reach the full potential of their technological exploitation. Among the different types of optical microresonators, ${ }^{[1]}$ especially surfacetension-induced whispering gallery mode (WGM) microresonators in the form of spheres ${ }^{[2]}$ and toroids, ${ }^{[3]}$ are the focus of interest, regarding their compact shapes with atomically smooth surfaces which enable the highest temporal and spatial confinement of light in terms of quality factor $\left(Q \leq 10^{9}\right)$ and mode volume, reducing the threshold for nonlinear optical interactions such as third harmonic generation, ${ }^{[4]}$ four wave mixing, ${ }^{[5]}$ and Raman lasing. ${ }^{[6]}$ Utilization of these appealing features has resulted in myriad applications including low threshold lasers, ${ }^{[7]}$ frequency comb generators, ${ }^{\left[{ }^{[8]}\right.}$ and extremely sensitive biological sensors. ${ }^{[9]}$ However, spherical WGM resonators have limited functionality due to the restriction of their on-chip integration by shape and a priori unknown eccentricity. Resonators with a definite state of eccentricity can be obtained by deforming a sphere between two solid plates, compromising spherical symmetry. ${ }^{[10]}$ A current state-of-the-art technology is the toroidal silica microresonator, produced by lithography and high power laser reflow techniques, which seem to have an advantage for mass production and on-chip integration. Unfortunately, the production demands individual surface reflow for each and every resonator with high temperatures (above $1000{ }^{\circ} \mathrm{C}$ ), hindering the integration of other optical components on the same substrate. ${ }^{[11]}$ In addition, using silica as a resonator material makes optical coupling to an on-chip high refractive

O. Aktas, E. Ozgur, Dr. O. Tobail, M. Kanik, E. Huseyinoglu, Prof. M. Bayindir

UNAM-National Nanotechnology Research Center Bilkent University

06800, Ankara, Turkey

E-mail: bayindir@nano.org.tr

E. Ozgur, M. Kanik, E. Huseyinoglu, Prof. M. Bayindir

Institute of Materials Science and Nanotechnology

Bilkent University

06800, Ankara, Turkey

O. Aktas, Prof. M. Bayindir

Department of Physics

Bilkent University

06800, Ankara, Turkey

Dr. O. Tobail

Egypt Nanotechnology Center

Cairo University

12588, Giza, Egypt

DOI: 10.1002/adom.201400072 index waveguide highly inefficient. Alternatively, chalcogenides have recently been considered as a material for active and passive resonant media because of their high refractive index $(n=2.2-3.4)$, wide mid-IR transparency window, extraordinary high nonlinearity, photosensitivity, low two-photon absorption, low phonon energy, and ability to be doped by most of the rare earth elements. ${ }^{[12,13]}$ Even though their Q-factors $\left(<7 \times 10^{7}\right)$ are not as high as that of silica WGM resonators, thresholds for some nonlinear optical interactions are on the same order of magnitude, due to their high nonlinearity. ${ }^{[14]}$ Nevertheless, similar obstacles exist before the full utilization of the chalcogenide microresonators, emerging from the exclusive nature of their production and integration phases. Methods compatible to mass production can produce hundreds of polydisperse spheres. ${ }^{[15,16]}$ However, spheres with a desired shape and optical quality need to be separated from the debris by very elaborate and laborious techniques, and manipulated by their attachment on a fiber tip ${ }^{[17]}$ or using optical tweezers. ${ }^{[18]}$ Other methods, similar to production of silica spheres, rely on melting the tip of a chalcogenide fiber by laser heating. ${ }^{[19]}$ Although these spheres are attached to a fiber stem, allowing them to be easily manipulated, high yield production is not possible due to the very nature of the process and the eccentricity caused by the stem. ${ }^{[20]}$ Besides spheres on a fiber tip, an additional example for the in-situ formation of WGM resonators is photoinduced microcavity resonators in chalcogenide microfibers. ${ }^{[21,22]}$ The integration of a single chalcogenide sphere coupled to a tapered fiber has been demonstrated by packaging the system using a UV-curable polymer. ${ }^{[23]}$ Another route towards the production of chalcogenide microspheres is to induce the Plateau-Rayleigh (PR) capillary instability ${ }^{[24,25]}$ in a chalcogenide fiber, which was first shown by optical fusing the bare core in the midair ${ }^{[16]}$ and recently in a polymer cladding for small fiber lengths $(<1 \mathrm{~mm})$ by using a tapering process ${ }^{[26]}$ and a local heat treatment. ${ }^{[27]}$

Here, we report a novel versatile method for the high yield production and on-chip integration of self-assembled globally oriented high- $Q$ WGM chalcogenide microresonators with surface-tension-induced spherical, spheroidal, and ellipsoidal boundaries with sub-nanometer roughness. The production involves the formation of chalcogenide microspheres within a polymer fiber of extensive length $(\geq 5 \mathrm{~cm})$, which was accomplished by preserving the integrity of the polymer cladding during thermal treatment. The transformation of these spherically symmetric resonators en masse into axisymmetric or asymmetric resonators was conducted by controlled plastic deformation. By using a special polymer as an adhesive layer in the integration phase, we demonstrate the near-perfect transfer and attachment of the microresonators embedded 
in the polymer fiber on any substrate, preserving their initial symmetries. As a result, novel on-chip chalcogenide WGM resonators are introduced to the WGM microresonator family. By optical characterization of the spherical and spheroidal microresonators, we routinely obtain very high $Q$-factors: up to $Q_{L}=3.1 \times 10^{5}$ which is, to our knowledge, the highest loaded $Q$-factor ever measured in $\mathrm{As}_{2} \mathrm{Se}_{3}$ WGM microresonators evanescently coupled to a silica tapered fiber.

For the production of chalcogenide WGM microresonators, multimaterial fibers, which consist of a chalcogenide glass $\left(\mathrm{As}_{2} \mathrm{Se}_{3}\right)$ core and a thermoplastic polymer polyethersulfone (PES) cladding, were used in this study. Fibers were obtained as a result of the size reduction of a preform (see Figure S1a) by thermal drawing in a fiber tower (see Figure S1b). The amorphous $\mathrm{As}_{2} \mathrm{Se}_{3}$ rod used in the production of the preform was prepared from high purity As and Se elements using a sealedampule melt-quenching technique (see the Supporting Information). ${ }^{[28]}$ As a result of fiber drawing, we obtained fibers (see Figure S1c) with different diameters, $d$, ranging from $1 \mathrm{~mm}$ to $30 \mu \mathrm{m}$, corresponding to $\mathrm{As}_{2} \mathrm{Se}_{3}$ core diameters ranging from $200 \mu \mathrm{m}$ to $6 \mu \mathrm{m}$. In order to produce a long chain of uniform $\mathrm{As}_{2} \mathrm{Se}_{3}$ microspheres embedded in a PES fiber, we developed a novel thermal treatment technique, which is based on extensive convective radial heating of the fiber with a conformal polymer cover preserving the integrity and straightness of the cladding at elevated temperatures (see Figure S2). A long uncovered fiber with free ends on a hot plate was observed to be bent or even twisted while releasing the built-in tension originating from the thermal drawing process.

On the contrary to the processing conditions to which a fiber is exposed during fiber drawing, such as sudden cooling under tension, thermal treatment of $\mathrm{As}_{2} \mathrm{Se}_{3}$ core PES cladding fiber at elevated temperatures $\left(260-310{ }^{\circ} \mathrm{C}\right)$ for substantial times leads ultimately to break-up of the continuous core into a chain of self-assembled spheres and inter-sphere satellites, due to overwhelming of surface tension against viscous forces. In order to understand the dynamics of PR instability and the evolution of the fiber core, we conducted finite element simulations (see the Supporting Information) by using temperature dependent viscosities for both materials (see Figure S3). Simulation and experimental snapshots of in-fiber microsphere formation (Figure 1a,b) reveal that amplitude of the dominant sinusoidal modulation on the core surface grows over time until pinchoff, at which point detachment occurs, leaving a smaller structure in the middle exposed to the same instability over and over again, resulting in a fractal pattern of main spheres with satellite spheres on their sides (Movie S1 and Movie S2). Satellite and sub-satellite sphere formations can be observed down to the $5^{\text {th }}$ generation, where the fractal process eventually stops reaching submicrometer scales (see Figure S4). Instability wavelengths, which are spatial periods of the structural perturbations on the fiber surface, determining the separation between the largest spheres at fiber core break-up, are given as a function of temperature along with the experimental and theoretical comparisons, and characteristic times for core break-up are given in Figure S5 and Figure S6, respectively. Pre-compensating the fiber diameter or adjusting the ratio of core diameter to outer diameter, a wide range of sphere sizes (1 mm-1 $\mu \mathrm{m})$ can be obtained. Fibers enclosing spherical a
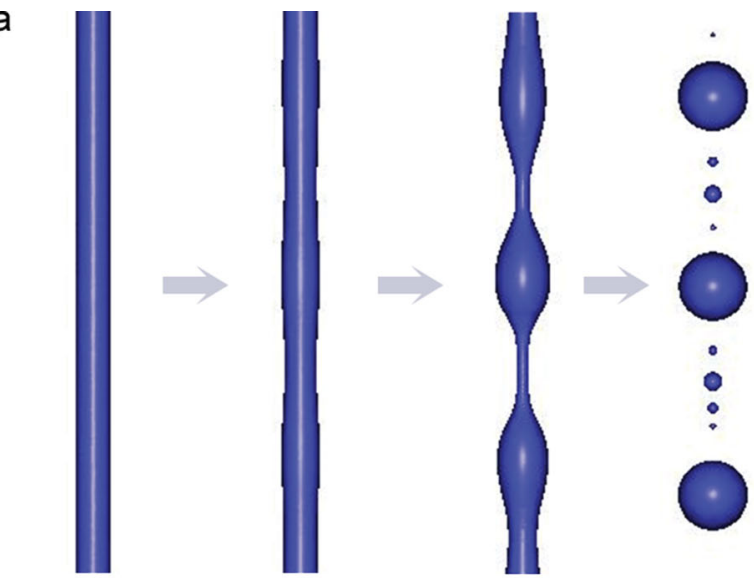

0

5

Time (min)

10
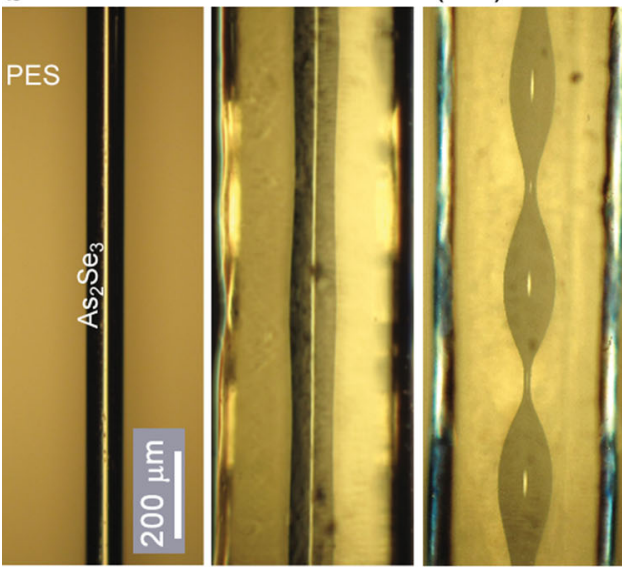

15

C

Figure 1. Simulation and experimental snapshots showing the evolution of microsphere formation in the $\mathrm{As}_{2} \mathrm{Se}_{3}$ core of a PES cladding fiber by Plateau-Rayleigh (PR) instability. (a) A finite element fluid dynamics simulation discloses the dynamics of PR instability occurring in the fiber. (b) Initially intact $80 \mu \mathrm{m}$ diameter chalcogenide core of the polymer cladding fiber turns into a self-assembled chain of $160 \mu \mathrm{m}$ diameter spheres and smaller satellite spheres embedded inside the fiber, in $15 \mathrm{~min}$ at $300^{\circ} \mathrm{C}$. (c) A photograph of a $5 \mathrm{~cm}$ long PES cladding fiber with embedded $\mathrm{As}_{2} \mathrm{Se}_{3}$ microspheres.

microresonators were produced as long as $5 \mathrm{~cm}$ in length, as shown in Figure 1c, limited only by the length of the tubular oven. However, uneven distribution of temperature or of stress caused by the conformal cover can result in unequal separations between main spheres.

Using continuous volume preserving transformations of the spherical microresonators induced by controlled plastic deformations in a custom made setup, we produced 3D ellipsoidal asymmetric resonant cavities (ARCs) with arbitrary eccentricity. ARCs, intrinsically possessing emission directionality, are important for laser applications ${ }^{[29]}$ as well as for fundamental studies of classical and quantum chaos, due to the resemblance between the Hamiltonian dynamics of a point mass moving 
in a $3 \mathrm{D}$ space constrained by hard walls, and the ray dynamics of the light in a uniform dielectric cavity. ${ }^{[30]}$ Also, recently, enhanced energy storage in deformed optical resonators was reported. ${ }^{[31]}$

Mechanical deformation of a PES polymer fiber enclosing an $\mathrm{As}_{2} \mathrm{Se}_{3}$ microsphere array between two parallel glass plates at a temperature of $280{ }^{\circ} \mathrm{C}$, which is above the glass transitions of the $\mathrm{As}_{2} \mathrm{Se}_{3}\left(T_{\mathrm{g}}=170{ }^{\circ} \mathrm{C}\right)$ and $\operatorname{PES}\left(T_{\mathrm{g}}=220^{\circ} \mathrm{C}\right)$, transforms the array of spheres into an array of triaxial ellipsoids, and finally into an array of "cigar-shaped" bodies globally oriented in a perpendicular direction to the fiber axis, because the fiber yields readily in this direction. Schematics of the fiber deformation process between two parallel glass plates in a high temperature furnace and deformation setup can be seen in Figure 2a and Figure S7, respectively. Optical reflection micrographs of deformed fibers can be seen in Figure $2 b-d$. The specular Fresnel reflection $(R=22 \%)$ from the surface of resonators can be used to easily discriminate spherical and ellipsoidal cavities. We used dichloromethane (DCM) to dissolve PES cladding and to reveal spherical, ellipsoidal and "cigar-shape" cavities. Scanning electron microscopy (SEM) micrographs of the resonators given in Figure $2 \mathrm{e}-\mathrm{g}$ show that it is possible to obtain smooth surfaces after deformations in a polymer encapsulation. SEM micrographs in Figure $2 \mathrm{~h}$,i show profile views of ellipsoidal and "cigar shape" cavities. Furthermore, we conducted atomic force microscopy measurements for the quantitative surface characterization of the microresonators. Sub-nanometer rms surface roughness $(\sigma<0.6 \mathrm{~nm})$ was found from a $500 \mathrm{~nm} \times 500 \mathrm{~nm}$ surface scan on top polar region of an ellipsoid (see Figure $2 \mathrm{j}$ ).

High yield production of spherical and ellipsoidal resonators inherently ordered in a polymer encapsulation can provide a unique advantage for multiple uses of these microresonators in photonics circuits, which is a critical barrier impeding their further development for relevant applications. Exploiting this advantage, we developed a method enabling on-chip integration of the chalcogenide microresonators with various shapes and sizes (see Figure 3a). In this method, the integration process involves two steps, which are the preparation of the substrate and the fiber encapsulation of an array of microresonators. We used $100 \mu \mathrm{m}$ thick glass coverslips as substrates, though there is no restriction for the substrate material. The substrates were spin coated with poly(vinylidenefluorideco-trifluoroethylene) P(VDF-co-TrFE) (see

a

Experimental Section). After numerous trials with different polymers, $\mathrm{P}(\mathrm{VDF}-\mathrm{co}$-TrFE) was found to be the most convenient as an adhesion layer regarding its low glass transition temperature $\left(T_{\mathrm{g}}=80^{\circ} \mathrm{C}\right)$, high adhesive forces towards chalcogenides, and
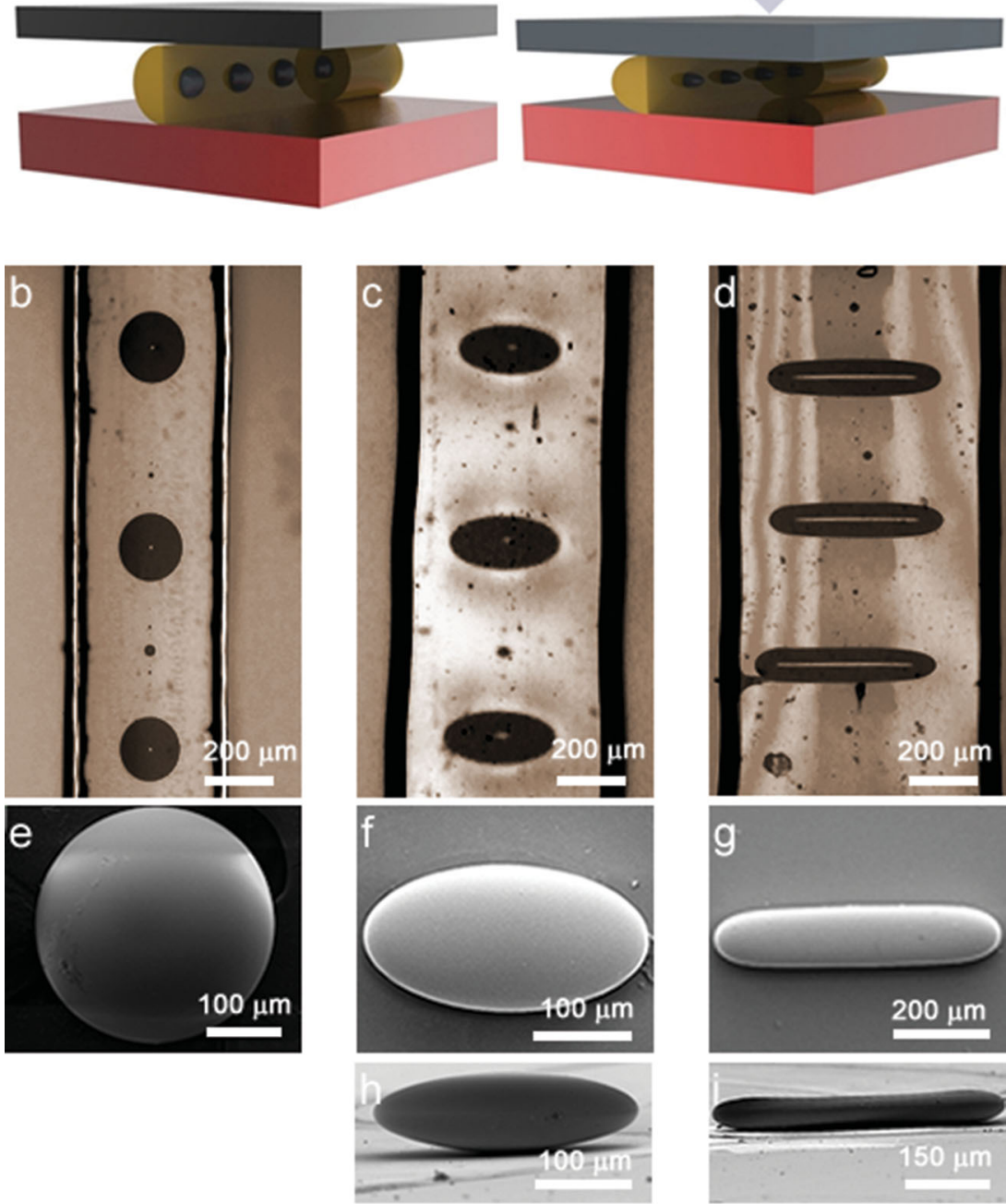

g
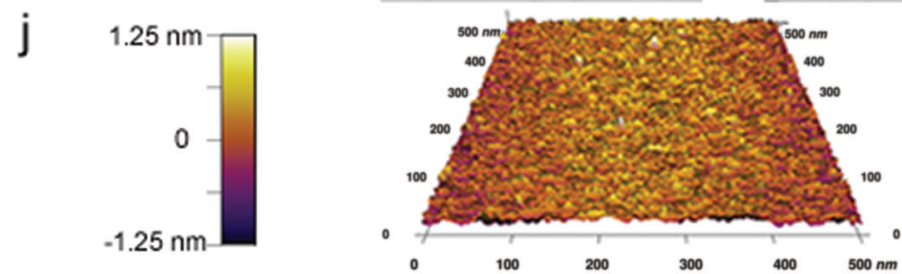

Figure 2. High yield production of 3D asymmetric microresonators via continuous volume preserving transformations induced by controlled plastic deformations. (a) Schematics of fiber deformation between two parallel glass plates in a high temperature furnace. Mechanical deformation of a PES polymer fiber enclosing an $\mathrm{As}_{2} \mathrm{Se}_{3}$ microsphere array at a temperature above the $T_{\mathrm{g}}$ of the both materials, transforms (b) the array of spheres into (c) an array of triaxial ellipsoids, then into (d) an array of "cigar-shaped" bodies globally oriented in perpendicular direction to the fiber axis. SEM micrographs show (e) spherical, (f) ellipsoidal, and (g) "cigar-shape" cavities extracted out by dissolving the PES polymer cladding in DCM. SEM micrographs show (h) ellipsoidal and (i) "cigar-shape" cavities in profile view. (j) AFM surface characterization of an ellipsoidal microresonator on $(500 \mathrm{~nm} \times 500 \mathrm{~nm})$ top polar region shows sub-nanometer rms surface roughness $(\sigma<0.6 \mathrm{~nm})$. 
a $1 . \mathrm{As}_{2} \mathrm{Se}_{3}$ core and PES cladding fiber

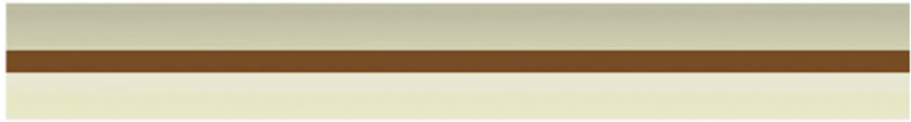

2. In-fiber microsphere cavity formation

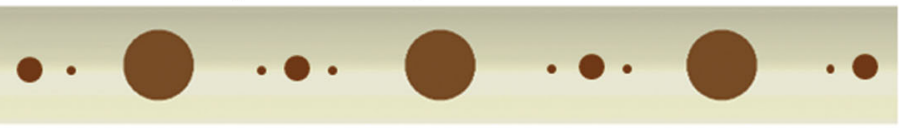

3. One side abrasion of the fiber

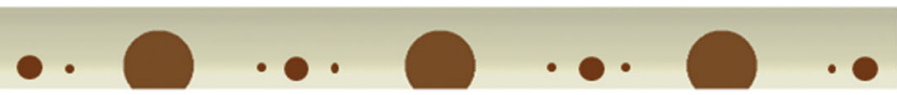

4. Transfer of the fiber on a substrate

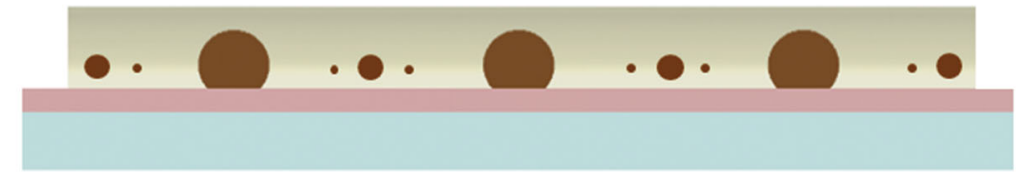

5. Selective dissolution of polymer encapsulation
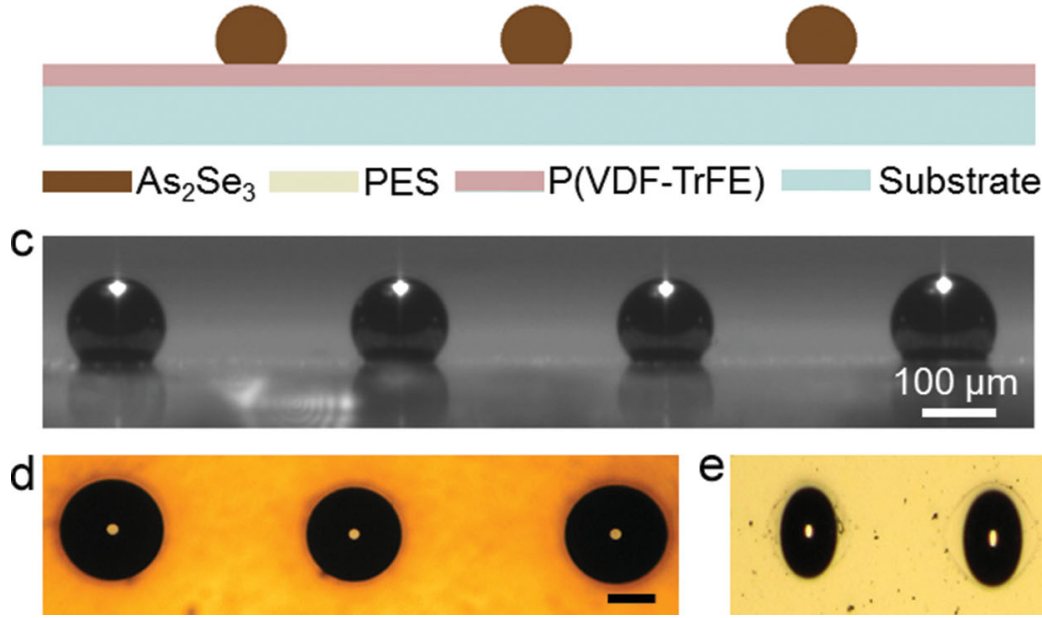

Figure 3. High yield production and on-chip integration of chalcogenide WGM microresonators on an arbitrary substrate. (a) The process starts with fabrication of $\mathrm{As}_{2} \mathrm{Se}_{3}$ core PES cladding fibers by thermal drawing. Then, in-fiber microsphere formation is induced by PR instability at elevated temperatures. As a third step, partial abrasion of the fiber cladding is achieved by a simple sandpapering process, exposing bottom sides of the spheres as contact surfaces for adhesion. In the fourth step, the resulting fiber is transferred manually onto a substrate spin coated with P(VDF-co-TrFE) and then heated to promote adhesion up to $210^{\circ} \mathrm{C}$, which is below the $T_{\mathrm{g}}$ of PES cladding but above the $T_{\mathrm{g}}$ of both As $\mathrm{Se}_{3}$ core and the P(VDF-co-TrFE) coating. At the last step, the largest microcavities attached to the surface can be released from the encapsulating PES polymer by selective dissolution in DCM, which has minimal effect on the substrate polymer coating. All satellite spheres are also flushed away spontaneously by the dissolution process. Optical micrographs show the (b) top, side and bottom views of the fiber after sandpapering one of its sides, (c) on-chip spherical chalcogenide microresonator array, (d) spherical microresonators directly integrated on the gold coated surface without any polymer coating, and (e) ellipsoidal microresonators integrated on P(VDF-co-TrFE) polymer coated surface. All scale bars are $100 \mu \mathrm{m}$.

chemical resistance against DCM. As for the preparation of the fiber, partial abrasion of the cladding was achieved by a simple sandpapering process (see Experimental Section), exposing the bottoms of the embedded microspheres as contact surfaces for adhesion. Optical micrographs of top, side, and bottom views of the fiber after sandpapering operation can be seen in Figure $3 b$. The resulting fiber was transferred manually onto the substrate spin coated with a P(VDF-co-TrFE) polymer and then heated to promote adhesion up to a temperature of $210^{\circ} \mathrm{C}$, which is below the $T_{\mathrm{g}}$ of the PES cladding, but above the $T_{\mathrm{g}}$ of the $\mathrm{As}_{2} \mathrm{Se}_{3}$ core and the P(VDF-co-TrFE) coating. Finally, microresonators with their bottoms attached to the substrate surface were revealed by selective dissolution of the encapsulating PES polymer in DCM (see Experimental Section). During the dissolution process all satellite cavities, which are smaller than the main cavities, are also flushed away spontaneously. This integration method enables the transfer of cavities on any substrate without any shape distortion, preserving the initial symmetry, due to protection by rigid PES encapsulation. An optical micrograph of on-chip well-ordered spherical chalcogenide WGM microresonator can 
be seen in Figure 3c. Integration of spherical microresonators directly onto the metal surfaces is also possible (see Figure 3d); however, it requires temperatures higher than the $T_{\mathrm{g}}$ of PES, which is not suitable for non-spherical cavities due to the softening of PES encapsulation, and surface tension compromising the non-spherical symmetry at elevated temperatures. Ellipsoidal microresonators integrated on a P(VDF-co-TrFE) polymer coated surface can be also seen in Figure 3e. Energy dispersive X-ray spectroscopy (EDX) results obtained from the surfaces of on-chip microsphere resonators demonstrate the consistence of the atomic ratio of $\mathrm{As}_{2} \mathrm{Se}_{3}$ (see Figure S8), besides showing that no impurity or residual polymer exists on the surfaces of microspheres after the dissolution process.

Another microcavity type with a different symmetry can be produced by compressing the on-chip microspheres upside down on a hot plate after their integration. Because of the different boundary conditions compared to those inside a polymer fiber, axisymmetric plastic deformation is favorable in this case and spheroidal microresonators with arbitrary elliptic cross sections can be easily produced. SEM micrographs of on-chip spherical/spheroidal/ellipsoidal chalcogenide WGM microresonator arrays are shown in Figure $4 a-c$.

We used tapered silica fibers (see the Supporting Information) with submicrometer waist diameters ( 700 nm) to evanescently couple light into these resonators and to capture transmission mode spectra (Figure $5 \mathrm{a}-\mathrm{d}$ ). Schematics of the experimental setup used for optical characterizations of microresonators is given in Figure S9. In order to eliminate thermo-optic effects (see Figure S10), we used very low optical input powers $(\sim 100 \mathrm{nW})$. Wavelength scanning ranges of the external cavity laser were set as $12 \mathrm{~nm}$ and $50 \mathrm{pm}$ for the acquisition of two free spectral range (FSR) wide spectra and single mode wide spectra, respectively. An adjustable polarization controller was used to maximize optical coupling into TE modes.

Despite the refractive index mismatch between the silica tapered fiber $(n=1.44)$ and the chalcogenide microresonators $(n=2.83)$ in the wavelength range of interest, it was possible to observe optical couplings to spherical and spheroidal microresonators, facilitated by a tapered silica fiber with sub-micrometer waist diameter, and resonators with small radii $(\sim 25 \mu \mathrm{m})$. The strength of the optical coupling critically depends on two parameters: the amount of field overlap, and phase matching between the fiber modes and the WGMs. ${ }^{[32]}$ Excitation of modes with high radial mode numbers are more favorable than low order modes in our case, because high order radial modes have low propagation constants, reducing phase mismatch, and higher evanescent field fraction outside the cavity, enhancing the field overlap (see the Supporting Information, including Figure S11). Reducing the size of the cavities also enhances optical coupling, which is in accordance with our experiments on microresonators under $60 \mu \mathrm{m}$ in diameter. We observed a series of resonance dips corresponding to WGMs of a microsphere $(D=50 \mu \mathrm{m})$ and an oblate microspheroid $(D=57 \mu \mathrm{m})$ with transmissions as low as $10 \mathrm{~dB}$. Resonance mode splitting can also be seen in the spectra of a microspheroid (see Figure $5 \mathrm{c}$ ), because of the broken degeneracy of azimuthal modes. In single mode spectra, a full width at half maximum value (FWHM) of $4.9 \mathrm{pm}$ was obtained by a Lorentzian fit to the resonance mode of the microsphere at $1551.858 \mathrm{~nm}$, corresponding to a loaded quality factor of $Q_{L}=3.1 \times 10^{5}$ associated with a transmission depth of $K=4.6 \mathrm{~dB}$ (see Figure $5 \mathrm{~b}$ ). The average of the loaded quality
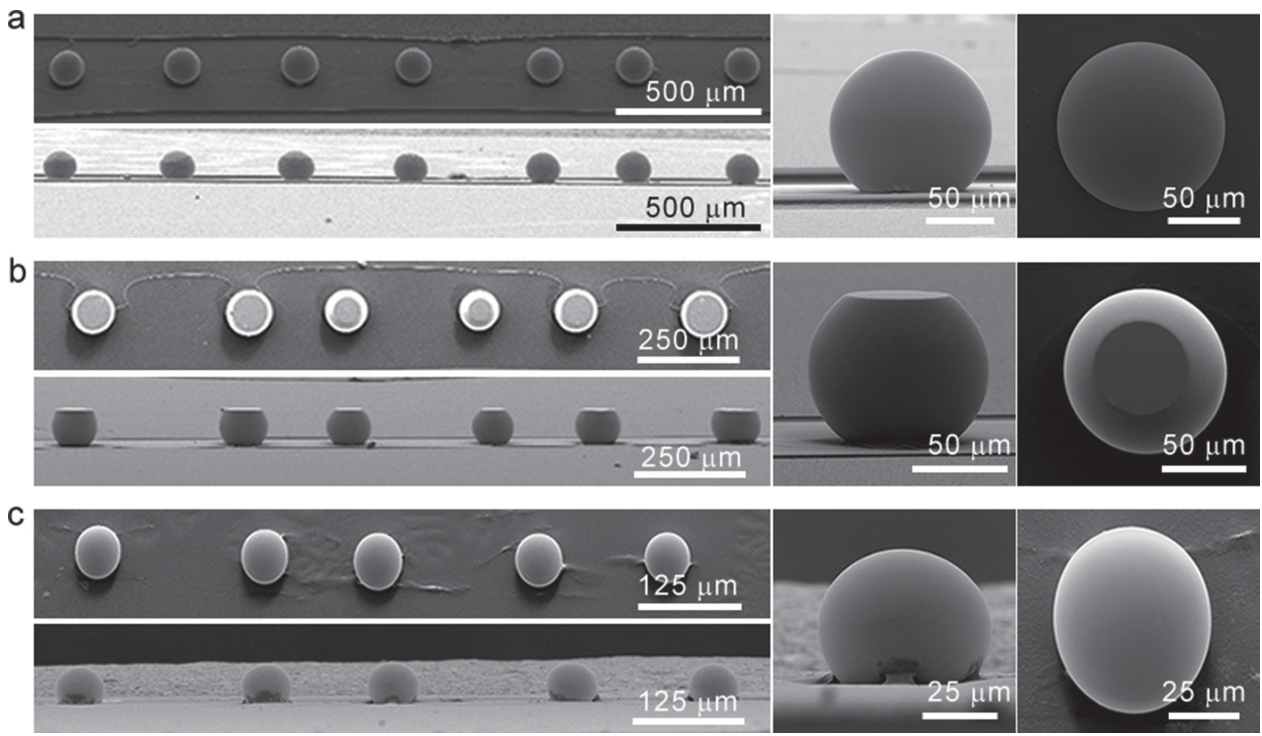

Figure 4. SEM micrographs of on-chip spherical/spheroidal/ellipsoidal chalcogenide microresonator arrays. Close-ups of some individual resonators in top and profile perspectives, show quality of production and on-chip integration such as the alignment of characteristic features (like equator plane), eccentricity, smoothness, and cleanliness of the resonator surfaces. (a) Top and profile SEM micrographs of spherical $\mathrm{As}_{2} \mathrm{Se}_{3}$ microresonator array and a single microsphere. Average diameter of spheres is $d_{\text {ave }}=124.4 \mu \mathrm{m}$ with standard deviation $\sigma=3.4 \mu \mathrm{m}(2.7 \%)$. As can be seen in the profile of the resonator, transfer and integration of the microsphere is accomplished with a very low aspherical deformation, and a good degree of parallel alignment of the equator plane with respect to the substrate surface. Top and profile SEM micrographs of (b) a spheroidal $\mathrm{As}_{2} \mathrm{Se}_{3}$ microresonator array and a single microspheroid, and (c) an ellipsoidal $\mathrm{As}_{2} \mathrm{Se}_{3}$ microresonator array and a single microellipsoid. 

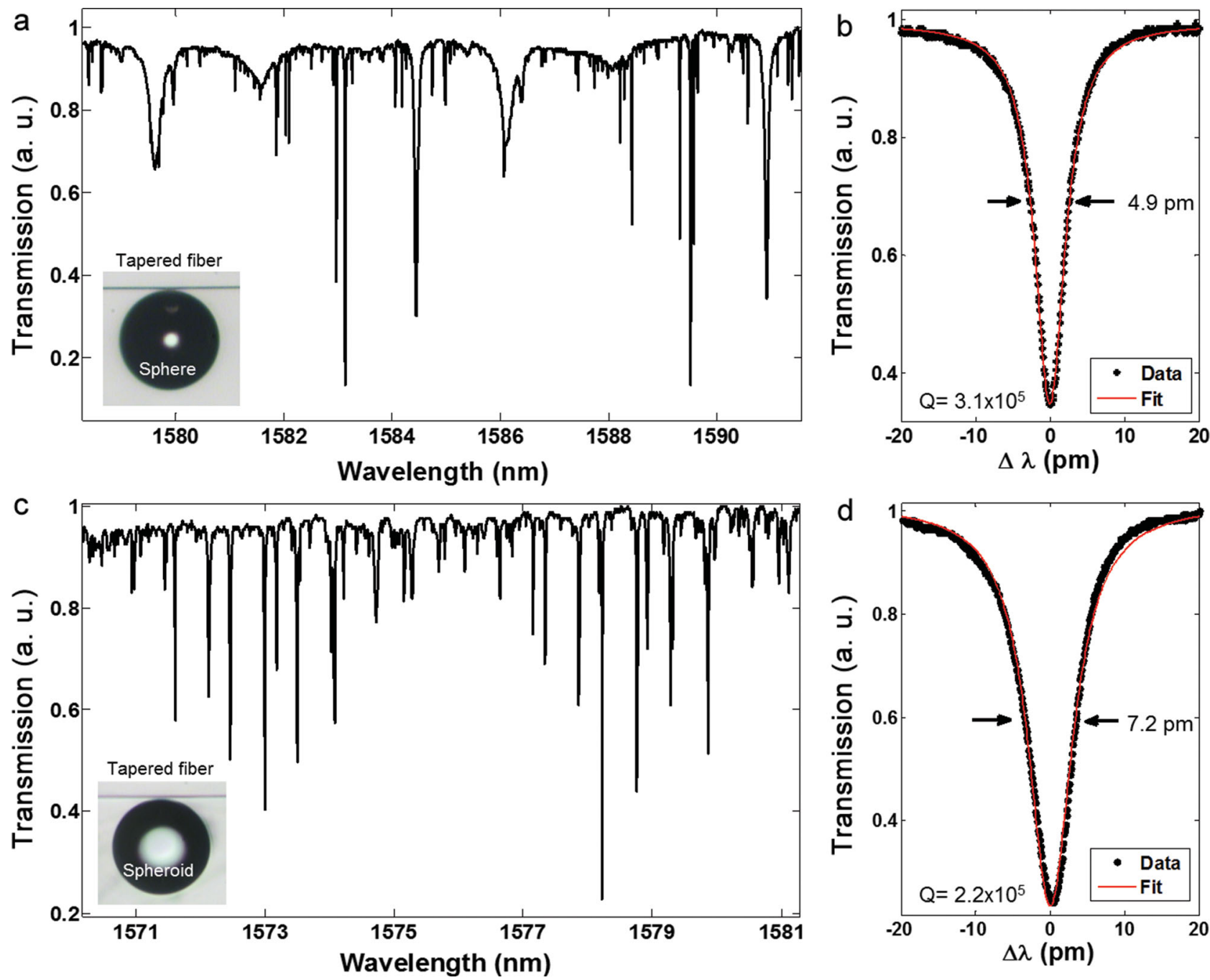

Figure 5. Optical characterizations of on-chip spherical and spheroidal chalcogenide microresonators. (a) Transmission spectra of an $\mathrm{As}_{2} \mathrm{Se}_{3}$ microsphere resonator of $50 \mu \mathrm{m}$ in diameter. Coupling strength of resonance modes can be as high as $10 \mathrm{~dB}$. FSR is $6.39 \mathrm{~nm}$. (b) Lorentzian fit to a resonance dip at $1551.858 \mathrm{~nm}$ shows that the FWHM and loaded quality factor $Q_{L}$ of the resonance mode are $4.9 \mathrm{pm}$ and $3.1 \times 10^{5}$, respectively. Inset shows evanescent coupling of light into the microsphere resonator using a tapered silica fiber with a sub-micrometer waist diameter. (c) Transmission spectra of an $\mathrm{As}_{2} \mathrm{Se}_{3}$ microspheroid resonator of $57 \mu \mathrm{m}$ in equator diameter. FSR is $5.22 \mathrm{~nm}$. (d) Lorentzian fit to a resonance dip at $1571.589 \mathrm{~nm}$ shows that FWHM and loaded quality factor $Q_{L}$ of resonance mode are $7.2 \mathrm{pm}$ and $2.2 \times 10^{5}$, respectively. Inset shows evanescent coupling of light into the microspheroid resonator using a tapered silica fiber.

factors we measured is $Q_{\text {ave }}=2.9 \times 10^{5}$ with a standard deviation of $\sigma=0.6 \times 10^{5}$. Similarly, a FWHM of $7.2 \mathrm{pm}$ was obtained by a Lorentzian fit to the resonance mode of the microspheroid at $1571.589 \mathrm{~nm}$, corresponding to a loaded quality factor of $Q_{L}=2.2 \times 10^{5}$, associated with a transmission depth of $K=6.2 \mathrm{~dB}$ (see Figure $5 \mathrm{~d}$ ). The maximum $Q$-factor reported for $\mathrm{As}_{2} \mathrm{Se}_{3}$ WGM resonators is $Q_{\max }=2.3 \times 10^{6}$, which was measured using a phase matched silicon waveguide evanescently coupled to a microsphere produced on the tip of a fiber. ${ }^{[19]}$

Experimentally measured quality factors $Q_{L}$ are described by the expression

$1 / Q_{L}=1 / Q_{O}+1 / Q_{c}$

written in terms of intrinsic $Q_{0}$ and extrinsic $Q_{c}$ quality factors, which are determined by resonator related losses and coupling losses, respectively. The intrinsic quality factor of WGM modes of microresonators $Q_{0}^{-1}=Q_{\text {rad }}^{-1}+Q_{s s}^{-1}+Q_{m a t}^{-1}$ are determined by several factors such as radiative losses $Q_{\text {rad }}^{-1}$, scattering losses $Q_{s s}^{-1}$, due to surface roughness and contamination, and material absorption losses $Q_{\text {mat }}^{-1}$. For intermediate size resonators $(D \sim 50 \mu \mathrm{m})$ with sub-nanometer surface roughness $(\sigma<0.6 \mathrm{~nm})$ as in our case, and in the absence of surface contaminants, $Q$-factor is only limited by material losses and given as ${ }^{[33]}$

$Q_{m a t}=2 \pi n / \alpha \lambda$

where $\alpha$ is absorption coefficient, $n$ is refractive index and $\lambda$ is wavelength. Using a material absorption coefficient $\alpha=1.6 \mathrm{~m}^{-1}$ of a commercially available $\mathrm{As}_{2} \mathrm{Se}_{3}$ glass at $1550 \mathrm{~nm}$ (see the Supporting Information including Figure S12a), the absorption limited intrinsic $Q$-factor was found to be 
$Q_{\text {mat }}=7.2 \times 10^{6}$, which is the maximum limit for experimentally obtainable quality factors (see Figure S12b). In addition, all WGM modes of the cavity suffer from the optical coupling process as well. The amount of coupling loss depends on phase matching and the field overlap of modes determined by mode order, radius of the cavity, radius of the tapered fiber, and the air gap between them. ${ }^{[34]}$ By adjusting the air gap, the quality factor $Q_{c}$ set by coupling loss can be tuned to achieve different regimes of coupling, where transmission $T$ drops to zero $(K=1)$ at critical coupling. Using the measured loaded quality factor $Q_{L}=3.1 \times 10^{5}$ and the transmission depth $K=0.65$ in the following derived expression, ${ }^{[35]}$

$$
Q_{0}=2 Q_{L}[1+\sqrt{1-K}] / K
$$

we calculated the intrinsic quality factor as $Q_{0}=0.76 \times 10^{6}$, corresponding to an absorption coefficient $\alpha=15 \mathrm{~m}^{-1}$. It can be considered to be on the order of magnitude of absorption limited quality factor $Q_{\text {mat }}$ of $\mathrm{As}_{2} \mathrm{Se}_{3}$. The discrepancy is assumed to be caused by a higher optical absorption in our synthesized chalcogenide glass or water condensing on cavity surfaces in lab conditions, at which we observed one order of magnitude degradation in the $Q$-factors of chalcogenide microresonators held three weeks in lab conditions.

We could not detect any transmission dips in the mode spectrum of triaxial ellipsoids with tapered silica fibers favoring only coupling to high order modes; however, we can directly observe light coupling into the micro-ellipsoids by thermal imaging (see Movie S3). The reason for the absence of transmission dips in the spectrum of ellipsoids could be due to the expected complete suppression of high order modes in deformed resonators of high eccentricity ${ }^{[36]}$ or some mechanisms similar to Arnold diffusion in the phase space of 3D ARCs, resulting in $Q$-spoiling with refractive escape of light, ${ }^{[37]}$ which require further theoretical studies of ARCs in 3D. Nevertheless, phase matched waveguides can be used to couple light evanescently into low order modes of ellipsoidal microresonators to observe transmission dips.

In summary, we have developed a simple, scalable, and lithography-free method for the production and on-chip integration of high- $Q$ factor WGM chalcogenide microresonators with spherical, spheroidal, and ellipsoidal boundaries with subnanometer surface roughness. High yield, low cost production of hundreds of self-assembled chalcogenide microresonators was achieved inducing PR instability in extended lengths of a multimaterial fiber. Since PR instability is a well-established phenomenon, our production and on-chip integration scheme are not limited to $\mathrm{As}_{2} \mathrm{Se}_{3}$ and can be applied to other important optical materials including $\mathrm{As}_{2} \mathrm{~S}_{3}, \mathrm{Si}, \mathrm{Ge}$, and $\mathrm{SiO}_{2}$, which can be turned into microcavities inside suitable cladding materials. ${ }^{[38-40]}$ Furthermore, active chalcogenide resonators can be made by doping with rare earth elements for on-chip laser applications. Utilizing the shape preserving protection of the polymer encapsulation, near-perfect transfer of the embedded microresonators onto any substrate in a globally oriented fashion is demonstrated. We observed loaded $Q$-factors as high as $Q_{L}=3.1 \times 10^{5}$ in our on-chip microcavity resonators. To our knowledge, it is the highest $Q$-factor ever measured in $\mathrm{As}_{2} \mathrm{Se}_{3}$ microresonators with silica tapered fibers favoring optical cou- pling only to high order WGM modes. Easy on-chip integration of highly nonlinear high- $Q$ microresonators can pave the way for new or extended exploitation of photonic devices in applications such as mid-IR sensors for the detection of molecular fingerprints, frequency comb generators for the generation of ultra-pure microwaves, ultra-low threshold microlasers with emission directionality, electro-optical tunable filters or modulators for optical communications, and optical logic gates for all-optical processors.

\section{Experimental Section}

Spin Coating of the Substrates: Before the integration of microresonators, substrates were spin coated with $\mathrm{P}(\mathrm{VDF}-\mathrm{co}-\mathrm{TrFE})$ solution for $45 \mathrm{~s}$ at $6500 \mathrm{rpm}$. The solution was prepared by sonification of P(VDF-co-TrFE) (30 g) in dimethylformamide $(50 \mathrm{~mL})$. Substrates were then placed on a hotplate at $100^{\circ} \mathrm{C}$ for $1 \mathrm{~h}$ to accelerate solvent evaporation.

Sandpapering of Fibers: To facilitate sandpapering process, fibers were attached to glass plate with double sided bands, then exposed parts of the fibers were rubbed against sheets of $\mathrm{SiC}$ sandpapers with size of abrasive particles decreasing from $5 \mu \mathrm{m}$ to $1 \mu \mathrm{m}$. After sandpapering process, fibers were released from the glass plate and cleaned by sonification in isopropyl alcohol for $10 \mathrm{~min}$.

Dissolution of PES Encapsulation: In order to remove the PES encapsulation of the microresonators integrated onto a substrate, we flushed the substrate with fresh DCM until most of the PES cladding dissolved away, then the substrate was placed in a fresh DCM solution for $1 \mathrm{~h}$, and finally flushed over with fresh DCM again to remove dissolved polymer residues. As a final treatment, substrate with integrated microresonators was placed in a vacuum oven at $50^{\circ} \mathrm{C}$ for $2 \mathrm{~h}$ to evaporate residual DCM.

\section{Supporting Information}

Supporting Information is available from the Wiley Online Library or from the author.

\section{Acknowledgements}

We thank Pelin Toren for her help during the cleaning process of microresonators with organic solvents and valuable discussions. This work was partially supported by TUBITAK under the Project No. $110 \mathrm{M} 412$. The research leading to these results has received funding from the European Research Council under the European Union's Seventh Framework Programme (FP/2007-2013)/ERC Grant Agreement n. 307357. M. B. acknowledges partial support from the Turkish Academy of Sciences (TUBA).

Received: February 12, 2014

Revised: March 29, 2014

Published online: May 6, 2014

[1] K. J. Vahala, Nature 2003, 424, 839

[2] V. B. Braginsky, M. L. Gorodetsky, V. S. Ilchenko, Phys. Lett. A 1989, 137, 393.

[3] D. K. Armani, T. J. Kippenberg, S. M. Spillane, K. J. Vahala, Nature 2003, 421, 925 
[4] T. Carmon, K. J. Vahala, Nat. Phys. 2007, 3, 430.

[5] P. Del'Haye, A. Schliesser, O. Arcizet, T. Wilken, R. Holzwarth, T. J. Kippenberg, Nature 2007, 450, 1214.

[6] S. M. Spillane, T. J. Kippenberg, K. J. Vahala, Nature 2002, 415, 621.

[7] V. Sandoghdar, F. Treussart, J. Hare, V. Lefevre-Seguin, J.-M. Raimond, S. Haroche, Phys. Rev. A 1996, 54, R1777.

[8] T. J. Kippenberg, R. Holzwarth, S. A. Diddams, Science 2011, 332, 555.

[9] F. Vollmer, S. Arnold, D. Keng, Proc. Natl. Acad. Sci. USA 2008, 105, 20701.

[10] G. S. Murugan, J. S. Wilkinson, M. N. Zervas, Appl. Phys. Lett. 2012, 101, 071106.

[11] M. Hossein-Zadeh, K. J. Vahala, Opt. Exp. 2007, 15, 166.

[12] B. J. Eggleton, B. Luther-Davies, K. Richardson, Nat. Photon. 2011, $5,141$.

[13] V. G. Ta'eed, N. J. Baker, L. Fu, K. Finsterbusch, M. R. E. Lamont, D. J. Moss, H. C. Nguyen, B. J. Eggleton, D. Y. Choi, S. Madden, B. Luther-Davies, Opt. Exp. 2007, 15, 9205.

[14] F. Vanier, M. Rochette, N. Godbout, Y.-A. Peter, Opt. Lett. 2013, 38, 4966.

[15] G. R. Elliott, D. W. Hewak, G. S. Murugan, J. S. Wilkinson, Opt. Exp. 2007, 15, 17452.

[16] C. Grillet, S. N. Bian, E. C. Magi, B. J. Eggleton, Appl. Phys. Lett. 2008, 92, 171109

[17] G. R. Elliott, G. S. Murugan, J. S. Wilkinson, M. N. Zervas, D. W. Hewak, Opt. Exp. 2010, 18, 26720.

[18] L. Zhang, Y.-X. Wang, F. Zhang, R. O. Claus, J. Opt. Soc. Am. B 2006, 23, 1793

[19] D. H. Broaddus, M. A. Foster, I. H. Agha, J. T. Robinson, M. Lipson, A. L. Gaeta, Opt. Exp. 2009, 17, 5998.

[20] A. Mazzei, S. Gotzinger, L. de S. Menezes, V. Sandoghdar, O. Benson, Opt. Commun. 2005, 250, 428.

[21] A. L. Watts, N. Singh, C. G. Poulton, E. C. Magi, I. V. Kabakova, D. D. Hudson, B. J. Eggleton, J. Opt. Soc. Am. B 2013, 30, 3249.

[22] F. Luan, E. Magi, T. Gong, I. Kabakova, B. J. Eggleton, Opt. Lett. $2011,36,4761$.
[23] P. Wang, M. Ding, T. Lee, G. S. Murugan, L. Bo, Y. Semenova, Q. Wu, D. Hewak, G. Brambilla, G. Farrell, Appl. Phys. Lett. 2013 102, 131110

[24] S. Tomotika, Proc. R. Soc. Lond. A 1935, 150, 322

[25] L. Rayleigh, Proc. R. Soc. Lond. 1879, 29, 71.

[26] S. Shabahang, J. J. Kaufman, D. S. Deng, A. F. Abouraddy, Appl. Phys. Lett. 2011, 99, 161909.

[27] J. J. Kaufman, G. Tao, S. Shabahang, E.-H. Banaei, D. S. Deng, X. Liang S. G. Johnson, Y. Fink, A. F. Abouraddy, Nature 2012, 487, 463.

[28] A. F. Abouraddy, O. Shapira, M. Bayindir, J. Arnold, F. Sorin, D. S. Hinczewski, J. D. Joannopoulos, Y. Fink, Nat. Mater. 2006, 5, 532.

[29] X.-F. Jiang, Y.-F. Xiao, C.-L. Zou, L. He, C.-H. Dong, B.-B. Li, Y. Li, F.-W. Sun, L. Yang, Q. Gong, Adv. Mater. 2012, 24, OP260.

[30] J. U. Nockel, A. D. Stone, Nature 1997, 385, 45.

[31] C. Liu, A. D. Falco, D. Molinari, Y. Khan, B. S. Ooi, T. F. Krauss, A. Fratalocchi, Nat. Photon. 2013, 7, 473.

[32] M. J. Humphrey, E. Dale, A. T. Rosenberger, D. K. Bandy, Opt. Commun. 2007, 271, 124

[33] M. L. Gorodetsky, A. A. Savchenkov, V. S. Ilchenko, Opt. Lett. 1996 $21,453$.

[34] C.-L Zou, Y. Yang, C.-H. Dong, Y.-F. Xiao, X.-W. Wu, Z.-F. Han, G.-C. Guo, J. Opt. Soc. Am. B 2008, 25, 1895.

[35] M. L. Gorodetsky, V. S. Ilchenko, J. Opt. Soc. Am. B 1999, 16, 147.

[36] V. S. Ilchenko, M. L. Gorodetsky, X. S. Yao, L. Maleki, Opt. Lett. 2001, 26, 256.

[37] S. Lacey, H. Wang, D. H. Foster, J. U. Nockel, Phys. Rev. Lett. 2003 , 91, 033902

[38] J. Ballato, T. Hawkins, P. Foy, R. Stolen, B. Kokuoz, M. Ellison, C. McMillen, J. Reppert, A. M. Rao, M. Daw, S. Sharma, R. Shori, O. Stafsudd, R. R. Rice, D. R. Powers, Opt. Express 2008, 16, 18675.

[39] P. Wang, T. Lee, M. Ding, A. Dhar, T. Hawkins, P. Foy, Y. Semenova, Q. Wu, J. Sahu, G. Farrell, J. Ballato, and G. Brambilla, Opt. Lett. 2012, 37, 728

[40] A. Gumennik, L. Wei, G. Lestoquoy, A. M. Stolyarov, X. Jia, P. H. Rekemeyer, M. J. Smith, X. Liang, B. J.-B. Grena, S. G. Johnson, S. Gradecak, A. F. Abouraddy, J. D. Joannopoulos, Y. Fink, Nat. Commun. 2013, 4, 2216. 